\title{
Training in minimal access surgery in the United Kingdom
}

Published online: 15 July 2004

(C) Springer-Verlag Berlin / Heidelberg 2004

\begin{abstract}
In April 2004 junior members of the British Society for Gynaecological Endoscopy met in Gloucester to discuss training in miminal access surgery in the United Kingdom. The following letter is a consensus of the trainees' opinions.
\end{abstract}

Keywords Training - Trainees · Minimal access surgery

\section{Introduction}

On 15 and 16 April 2004 the fifth meeting of the British Society for Gynaecological Endoscopy (BSGE) senior trainees was held in Cheltenham. The aim of the meeting was to discuss training in minimal access surgery (MAS) in the United Kingdom to feed trainees' views into the evitable changes that are now and will be occurring over the next few years. The views expressed in this letter are a consensus view from those trainees at the meeting. Items discussed included (a) the integration of MAS training into the current Royal College of Obstetricians and Gynaecologists (RCOG) core and post-core training, (b) training in advanced MAS, (c) possible development of a master's degree in MAS, (d) possibility of gynaecology moving to office gynaecology and surgical gynaecology as separate areas.

\section{The integration of MAS training into the current RCOG core and post-core training}

The RCOG has now divided training into core training and post-core training. The former is the level of competence that all gynaecologists are expected to have

The author wrote this article on behalf of the BSGE trainees

T. T. Carpenter $(\bullet)$

Gynaecology Department,

Royal Surrey County Hospital,

Egerton Road, Guildford, Surrey, GU10 1AL, UK

e-mail: t.carpenter@ doctors.org.uk

Tel.: +44-7766-521970 across the field of gynaecology, and these should be obtained before the trainee progresses from year 3 to year 4 of the 5-year training scheme. Such a degree of competency should ensure that the gynaecologist is able to cover a general gynaecological on call and be able to deal with the emergency conditions that arise. Bearing this in mind, the trainees feel the levels required with in the core log book are too low, and that they should include up to and including laparoscopic management of ectopic pregnancy. The overall proportion of ectopic pregnancy that is managed laparoscopically in the UK remains pitifully small compared to that in the rest of Europe and in North America, and the fact that many new Certificate of Completion of Specialist Training (CCST) holders are still unable to perform this relatively basic procedure will do nothing to address this situation.

With regard to higher training in MAS the provisional special skills modules (SSM) in laparoscopic and hysteroscopic surgery were presented. The modules were developed by the RCOG in conjunction with the BSGE and follow the same format as the other SSMs developed by the college. The level of procedures undertaken in these modules correspond approximately to those in the old RCOG MAS level 2 procedures. There will be no formal list of preceptors; instead the trainee will select a trainer who is able to offer adequate training to complete the module and, if they accept, will be registered as the preceptor. There are some additional requirements (basic) that the preceptor must meet to be eligible which are outlined in the module. Overall these were welcomed by trainees. It is hoped these will be available from around the middle of this year.

\section{Training in advanced MAS}

Whilst the SSMs in MAS are welcomed, it is recognised that this is insufficient to produce MAS surgeons able to undertake advance procedures such as stage 4 endometriosis or laparoscopic sacrocolpopexy. Clearly some system needs to be in place to provide such training for 
some (albeit a relatively small number) who wish to obtain such training. At last year's meeting it was felt this would be best achieved by MAS fellowships. The structure suggested last year was that these would be of around 2 years duration with an agreed job plan in a BSGE approved training unit. It may well be necessary to move between trainers during this time to achieve an adequate breadth of experience. It may also be advantageous to be able to include training overseas in some of these posts. During this time research would be encouraged, and the possibility of undertaking a higher degree should be available. This would not be mandatory, however. The posts would have to be vetted by the BSGE committee to ensure they are of a high enough standard to maintain their fellowship status. The question of fellowships was again discussed and strongly supported by trainees. We would be keen to see the development of such schemes begin as soon as possible and feel it essential that there is trainee in put into the process as well as more senior members of the BSGE

Although such training may be possible as 'out of programme experience', on the whole it is becoming increasingly difficult to gain approval for such time out from the regional Specialist Training Committees and/or the Postgraduate Deans. As such it is probable that in most cases such fellowship may need to be undertaken post-CCST.

\section{Development of a masters degree in MAS}

At last year's meeting the idea of the development of a master's degree in MAS was muted by some members of the BSGE executive. It was suggested that this not only be available to trainees wishing to undertake higher level MAS but also may be a requirement for those preceptors who wish to teach advanced MAS. At the time such a proposal was not supported. It was felt that MAS is a surgical discipline for which training was best given and received in an apprenticeship style, and that the holding of a master's degree would not particularly improve one's ability to teach a very practical skill. Additionally, if such a qualification became mandatory, many ex- tremely good trainers would simply stop training rather than spending additional time and money to obtain the degree. Following further discussion on this topic at this year's meeting the trainees remain unenthusiastic about the development of a master's degree for all the reasons previously stated.

\section{Possibility of gynaecology separating into office gynaecology and surgical gynaecology as separate areas of interest}

With the decreasing amount of gynaecological surgery and the reducing time available for training there was concern that it is becoming increasingly difficult to obtain adequate experience in gynaecological surgery. Procedures and techniques are becoming more sophisticated all the time, and there is an increasing move to sub- specialisation. In much of the rest of Europe the specialty has largely developed into 'office' gynaecologists who undertake clinics, colposcopy, out-patient hysteroscopy etc. and the gynaecological 'surgeon'. The latter receives referrals from 'office' gynaecologists, performs the surgery and then passes the patient back for ongoing care. Such a model of care is also being piloted by the British urologists who will be awarded a CST at the end of year 3 and then will have to compete for higher training to allow them to undertake advanced urological surgery. Those who do not pursue this advanced training would simply refer the patients on if such surgery were necessary. Whilst this would be a dramatic change, the trainees generally feel that such a situation is inevitable in gynaecology if training is to be obtained and skills maintained by adequate through-put. Overall this may well improve care for patients, although it is accepted it may not be popular with the specialty as a whole.

In summary the trainees felt improved training could be best achieved by, (a) ensuring all trainees are competent to perform laparoscopic treatment of ectopic pregnancy by the end of core training, (b) the development of fellowships for advanced training in MAS and (c) the development of gynaecological 'surgeons'. 